\title{
A Standard Process for Sustainable Design
}

\author{
Francisco J. Farias Stipo * \\ Kay Elliott Architects, Torquay, Devon, TQ1 2JP, United Kingdom
}

\begin{abstract}
This exploratory research examined the degree of adoption and impact of the concepts of Building Information Model (BIM), Integrated Project Delivery (IPD), Integrated Design Process (IDP) and Building Energy Simulation (BES) on the design processes of advanced architectural firms when executing sustainable design. Six offices identified by the press and peers' recognition for a strong commitment to sustainable design and influence in the design of high performance buildings were selected. In semi-standardized interviews, these firms presented their perceptions of the influence of BIM, BES, and IPD/IDP. The results show that a generalization of sustainable design processes is possible. A design process for sustainability (DEPROSU) model was created by collecting best practices from data gathered from the interviews and the critical literature review. This research provides evidence of commonalities found in the design processes of the selected firms. These commonalities represented in the DEPROSU model can potentially be validated as protocols or standards for sustainable design, providing architectural design practices with concrete patterns for improvement and or validation of their design methods.
\end{abstract}

Keywords: sustainability; Building Information Modelling; building energy simulation; design processes; integrated design.

processes that together contribute to a new process for design of sustainable dwellings. This research endeavored to

\section{Introduction}

Architecture and buildings are identified as being of major importance in reducing the intensity of global warming and ameliorating the impacts on humanity [1], [2]. Existing research shows that the most important decisions occur at the earlier stages of design, having the greatest impact on the Life Cycle Cost (LCC) of the building [3]. Architecture firms are required to produce more energy efficient buildings [4]. The three emerging concepts of Building Information Modelling (BIM), Building Energy Simulation (BES) and Integrated Design Process (IDP) provide a new opportunity to address the challenges of achieving sustainable communities and ameliorating the impacts of global warming.

The American Institute of Architects (AIA) contracts still guide many design projects through a conventional and linear process through consecutive phases [5]. The hypothesis behind this research is that BIM, BES, and IDP are complementary determine to what extent leading firms are using a design process employing these three technologies and processes. The research collected data by interviewing designers at leading firms. The data analysis provided generalizations regarding contemporary design processes and achieves insights into new and appropriate design processes that make use of new technologies.

\section{Definitions}

Sustainable design: Ecodesigners tend to analyze the impact of a building using a holistic approach including LCC and Life Cycle Analysis (LCA) evaluations [6], [7] and ecological principles [8].

Integrated Design Process (IDP): IDP is a collaborative process with a multidisciplinary design team that focuses on the design, construction, operation and occupancy of a building over its complete life-cycle, with a clear definition of

* Corresponding author. Tel.: +44-755-206-0101

E-mail: francisco.farias@kayelliott.co.uk

(C) 2011 International Association for Sharing Knowledge and Sustainability

DOI: $10.5383 /$ ijtee.04.0x.00xx 
environmental and economic goals and objectives [9]. According to Zimmerman, the main components of the Integrated Design Process are:

1. Interdisciplinary work between designers, engineers, operations people, costing specialists, future occupants and other significant actors participating at the beginning of the design process;

2. The addition of a specialist in the field of sustainability, energy performance and comfort;

3. No separation between individual building systems in the total budget. Budget restrictions are set at the whole-building level;

4. Discussion of the importance of performance issues, and a consensus on building performance between the client and the architects;

5. Clear definition of the performance goals and strategies that will be updated throughout the design process;

6. The addition of subject specialists, such as daylighting, for specific consultations with the design team;

7. Development of various design alternatives that will be tested with energy simulation, to provide evidence-based design choices.

Kow \& Grondzik [10], state that IDP is "knowledge applied in parallel", which refers to a simultaneous interaction among stakeholders, while conventional design is "knowledge applied in series", which is a linear sequence of steps rather than simultaneous activities. According to the authors, the steps towards an IDP are: (1) establishing commitment; (2) team formation and setting goals; (3) information gathering; (4) conceptual/schematic design; (5) testing of design alternatives; (6) design development; (7) construction; and (8) assessment/verification (Facility Management and operations of the building).

BIM: BIM is a digital representation of physical and functional characteristics of a facility and serves as a shared source for information for it [11]. BIM software provides objects that represent architectonic elements, parametric 3D modeling, rendering functions, automated drafting, rich graphic and nongraphic information stores, and interoperability to analysis programs [12]. Although IPD is possible to achieve without using BIM, the AIA report on Integrated Project Delivery recommends BIM as an essential driver with the potential to support IDP activities [5]. BIM adoption is accelerating toward universality in the United States [13]. BIM tools penetrate more deeply in larger firms, with nearly $100 \%$ of firms that average 100 or more employees make use of BIM. One-third of the firms consulted in this study are also using BIM software to share models with consultants, and 29\% mentioned the use of BIM for 3D clash detection -also called 4D simulation, which enables designers to identify coordination errors with multiple consultants.

Smith \& Tardif [14] define education of professionals as the largest and often hidden investment for the cultural shift. From further education in the new methods, the profession would achieve greater value than by simply automating existing processes.
BES: Sustainable design processes usually rely upon BES software to establish expected energy consumption of building designs [15]. BES tools are best suited for examination of risk and to test design alternatives [16]. However, in spite of questionable accuracy and validity, they are often used to demonstrate that a particular design alternative is able to meet certain performance requirements.

\section{Research Methods}

An exploratory qualitative research approach was selected to investigate this research topic [17], which implemented critical literature reviews, case study analysis and interviews. Three offices in the U.S.A., two offices in the U.K. and one in Malaysia were selected due to their commitment to sustainable design and influence in the design of high performance buildings as identified by the press. Qualitative data was collected through semi-standardized interviews from design professionals to acquire insight into the interaction of BIM, IDP, and BES on sustainable design.

The open-ended interview questions were divided into different sections and presented to a particular individual with expertise in the area. When possible, multiple participants were interviewed simultaneously to provide a fluid discussion and to improve reliability of data. The interviews took place in the informant's offices. The number of total informants is summarized in Table 1. The informants included designers from different hierarchical levels and positions, as shown on Table 2. The inclusion of such variety depended on their availability and willingness to participate which was not equal across the offices and therefore, a comparison of roles across the different offices was not possible.

In general, each interview began by asking their familiarity with any of the concepts of sustainable design, BIM, IDP and BES. Then they were asked about the overall design process for high performance buildings and their use of BIM, IDP and BES. The interviews lasted in general one hour and the participation was voluntary -no compensation was provided. All recorded interviews were later transcribed verbatim and used as raw data for analysis and coding. The data was analyzed using transcription; coding; thematic analysis and exploratory analysis. The transcription of the interviews produced 49,979 words of data. To code the transcripts, a number was allocated to the responses according to the following a priori codes:

1. Use of BIM;

2. Use of BES;

3. Use of IDP;

4. Sustainable Design Workflow;

5. Other findings.

Internal validity was checked by using respondent feedback, to see if the interpretation mirrors their experience [18], but only two participants confirmed. 


\section{Traditional design process}

This section will discuss about the most influential delivery methods and best practices in the US, represented by the AIA protocols and documents, in addition to scholarly works about sustainable architecture.

\begin{tabular}{l|llllllll}
\multicolumn{2}{l}{$\begin{array}{l}\text { Table 1: Summary of Interview and Archival Data. } \\
\text { Offices }\end{array}$} & 1 & 2 & 3 & 4 & 5 & 6 & Total \\
\hline $\begin{array}{l}\text { No. interviews } \\
\text { No. informants }\end{array}$ & 1 & 3 & 1 & 1 & 1 & 1 & 8 \\
$\begin{array}{l}\text { No. pages } \\
\text { transcription }\end{array}$ & 30 & 26 & 15 & 22 & 17 & 29 & 139
\end{tabular}

Table 2: Key actors involved and their position in their respective firms.

\begin{tabular}{l|ll}
\multicolumn{1}{l|}{ OFFICES } & KEY ACTORS FUNCTION & BACKGROUND \\
\hline 1 & Sustainability Manager & Environment Management \\
& Associate Architect & Architecture \\
& BIM Manager & Technology \\
& Simulation/Integrated Design & Architecture \\
2 & Sustainability Coordinator & Mechanical Engineering \\
& Partner, Contract Supervisor & Architecture \\
3 & BIM Manager & Architecture \\
4 & Principal, Chief Sustainability Officer & Architecture \\
& Associate Principal, Senior V.P. & Architecture \\
& Sustainability Officer & Architecture \\
5 & Associate Architect & Architecture \\
& Architectural Assistant & Architecture \\
& Principal, Sustainability Chief & Architecture
\end{tabular}

According to the AIA best practices [5], the design process is divided into phases of schematic design (SD), design development (DD), construction documents, bid or negotiation and construction administration. In Schematic Design (SD), the architect consults with the owner to determine project goals and requirements. Often this determines the program for the project. Deliverables for SD often produces a site plan, floor plan(s), sections, an elevation, and other illustrative materials; computer images, renderings, or models. Typically the drawings include overall dimensions, and a construction cost is estimated. In the Design Development (DD) phase, designers use the initial design documents from the schematic phase and take them one step further. This phase lays out mechanical, electrical, plumbing, structural, and architectural details. Deliverables for DD often include floor plans, sections, and elevations with full dimensions. These drawings typically include door and window details and outline material specifications. In the Construction Document (CD) phase, once the owner and architect are satisfied with the documents produced during DD, the architect produces drawings with greater detail. The deliverables for $\mathrm{CD}$ are a set of drawings that include all pertinent information required for the contractor to price and build the project. In Bid or Negotiation phase, the first step is the preparation of the bid documents to go out to potential contractors for pricing. The deliverable is a construction contract. Once this document is signed, project construction can begin. In the Construction Administration (CA) phase, services are rendered at the owner's discretion and are outlined in the owner-architect construction agreement. Different owner-architect-contractor agreements require different levels of services on the architect's part. CA services begin with the initial contract for construction and terminate when the final certificate of payment is issued. The architect's core responsibility during this phase is to help the contractor to build the project as specified in the CDs as approved by the owner. The deliverable is a successfully built and contracted project.

This sequence presents a linear process where the major efforts are not front loaded and receive no multidisciplinary feedback from consultants. Without an IDP and without BIM, the risk of construction errors and poor building performance is significantly high. Traditionally, the AIA offered different project delivery methods which are widely used in the AEC industry and have an impact in the design such as [5]:

- Design-Build-Bid (DBB),

- Multiple Prime,

- Design Build under Best Value Selection with Bridging,

- Construction Manager at Risk, 
- Design-Build under Best Value Selection with Criteria,

- Design-Build under Qualification Based Selection and

- Integrated Project Delivery (IPD)

In some delivery methods like the Design-Build-Bid, the constructor is not even allowed to participate in the conceptualization of the project. General contractors bid on the project with the lowest qualified bidder being selected, which attempts to the high quality goal of the project. Moreover, the tension and conflicts among participants often results in higher costs from change-orders, repairs and lawsuits [5], [19].

Arguably as a consequence of both IDP and BIM capabilities, the front-loaded effort in an IDP creates a mismatch between the traditional and linear design method where the major efforts and consequent proportional fees are dedicated to the later stages.

\section{Emerging process in advanced firms}

Vallero \& Brasier [20] state that a sustainable design strategy needs to surpass the short-term economic benefits of traditional methods and see the design from a holistic viewpoint. The authors further differentiate two design models: the traditionallinear process, and the transitional model. The linear model tends to set as priority variables such as monetary costs, scheduling constraints and quality. The transitional model includes a green building rating system (GBRS) and moves the technical input earlier in the design process, having a higher level of interaction and feedback among all stakeholders. Other researchers studied the use of digital tools to aid sustainable design and analysis [21], [22], [23]. Therefore, contemporary architecture design is moving towards an emphasis on sustainability and reliance upon evidence-based design (including BES) to support design decisions [24], [25].

According to the data from the interviews, a design process for sustainability involves a series of steps clustered as: (a) programming, (b) site analysis, (c) goals definition, (d) analytics, (e) design refinement, (f) construction and (g) operations. However, this study focused on the implementation from (a) to (e). In general, the participants start with an analysis of the orientation, site and climate, as well as the urban or rural conditions. That information orients designers to choose specific passive design strategies. Simple massing simulation analysis is performed to compare design alternatives for schematic design and to refine building shape. When the design approaches the design development stage, most of the simulation is already done and the design is refined. The next step is for coordination using BIM and clash detection. BIM can be used for commissioning and FM, providing information about the building's performance and new lessons.

Participants seem to agree that a holistic approach is necessary for sustainable design, and that it depends upon fostering a multidisciplinary effort and accepting a radical change in processes [26]. Post-construction evaluations are currently not fully adopted, although some GBRS are requiring it. BIM's full potential is currently not achieved in most firms. BES is increasingly adopted in design process to predict and improve building performance. IDP is considered a critical aspect of a contemporary design model for sustainability and seems to have a synergetic effect on BIM and BES. The participants considered BIM to be the essential "vehicle" to facilitate communication and coordination between stakeholders by including IDP and BES.

Regarding the use of BIM from the participants:

- Given six offices, five have adopted BIM and have a strong commitment to BIM;

- Participants appreciate BIM capabilities to expedite design production and coordination;

- The appreciation for BIM is increased when used in conjunction with consultants;

- Interoperability remains a challenge;

- BIM standards remain a challenge;

- $\quad$ BIM has supported the implementation of BES;

Regarding the use of BES from the participants:

- Given six offices, six adopted BES in their design methods;

- Four out of six are implementing in-house energy simulation for early design stages;

- Six offices outsource BES for advanced design stages;

- BES use varies depending on projects' complexity and/or fee structure;

- During advanced design stages, firms usually outsource simulation;

- The use of a particular BIM tool can affect the choice of the simulation engine;

- High-end simulation such as CFD is used for complex buildings with a high fee structure.

Some firms have made attempts to standardize aspects or subprocesses of their designs methods for sustainability, and the commonalities found suggest that a generalization is possible. According to the participants, a challenge in developing a standard design method for sustainability is flexibility, where the method should enable a good fit to most design situations. The other challenge was related to the type of method. A performance-based method would encourage innovation and higher standards, while a prescriptive model would lead to mediocre results as best8. A combination of both would allow an easy to follow description of steps (prescriptive), while some sub-processes in each step would impose performancebased objectives (combining BIM with BES in an IDP).

\section{DEPROSU}

After analyzing data from the interviews and the literature [8], [27], the commonalities found have been synthesized into a process that could be standardized across firms, such as the Design Process for Sustainability (DEPROSU). DEPROSU is a prescriptive and performance-based flexible model of design that better reflects the reality observed in advanced design 
firms. It is tailored to a multidisciplinary design team (IDP) using BIM and BES proficiently.

The DEPROSU model comprehends three main phases; each containing two sub-phases A \& B. Every phase A represents an information gathering process in anticipation of phase $B$ (analysis and synthesis, respectively). A simplification of their process across the sample is defined in the following Figure 1. In Phase 1A the order of sub-processes is interchangeable without significantly affecting the final result.

\subsection{Phase 1A: Information gathering for Design}

Programming: In this step, a detailed description of the requirements for the design is done by specialists [28].

For automated processes, there are several software that helps organizing and evaluating programs, such as Trelligence Affinity (http://www.trelligence.com/) and dRofus ${ }^{\mathrm{TM}}$ (http://www.drofus.no/en/index.html).

In the highly accepted Problem Seeking method, Peña and Parshall [28] distinguish between the architectural programmers and design architects. Architectural programmers are responsible for developing a detailed description of needs and requirements, while design architects synthesize the proposed solution into a form to be evaluated. An alternative approach was created by The Whole Building Design Guide which proposes a six step for programming, defined as follows [29]:

1. Research the project type: the types of spaces, the space criteria, typical relationships of spaces for these functions, typical ratios of net assignable square footage to gross square footage, etc.

2. Establish goals and objectives: Organizational Goals, Form and Image Goals, Function Goals, Economic Goals, Time Goals and Management Goals.

3. Gather relevant information: in this step they analyze site conditions, topography, etc.

4. Identify strategies: By using "bubble" diagrams, you can indicate what functions should be near each other in order for the project to function smoothly. Some examples of common categories of programmatic strategies include:
a) Centralization and decentralization
b) Flexibility
c) Priorities and phasing
d) Levels of access
e) Determine quantitative requirements
f) Summarize the program

\subsubsection{Site}

During this step, several components and sub-components are identified. Climate will dictate fundamental characteristics of the site to be considered, such as heating and cooling degree days; solar orientation (passive heating/cooling) and operational system (passive mode; mixed mode; full mode; productive mode; and composite mode). To help analyzing climate and possible passive strategies, software like Climate
Consultant is recommended [30], along with Psychometric charts and windrose diagrams.

\subsubsection{Goals definition}

In this step, stakeholders define the Performance Goals and Measurement Criteria, and also establish the design team with an up-front loaded multidisciplinary work. They might decide to pursue a GBRS certification and a third party to account for achievements. For the measurement criteria, several protocols (ASHRAE, ISO, IESNA, EPA, DOE, Part L (U.K.)), third party certification teams and verification methods (postoccupancy analysis, building commissioning, BES) can serve to confirm that the goals were reached. The team building is arguably the most critical aspect of this step, where all design consultants (structural, MEP, etc.) are selected and the design atmosphere is set.

\subsection{Phase 1B: Design}

\subsubsection{Analytics:}

This step represents one instance of an iterative loop where all stakeholders and design consultants work together to specify design strategies. BIM adoption is highly recommended for a fluid communication among design consultants, being the central repository of data. Stakeholders define in probably several loops of discussion and analysis, the choice of MEP systems, passive technology, the environmental steer and several design alternatives represented by basic massing analyzed with BES tools. This process derives the final envelope design.

\subsubsection{Design Refinement}

The design team shall execute several iterations of analytics where design consultants' feedback exchange enhance and refine the design. It can include a detailed and/or advanced energy simulation. BIM is again critical for a fluid communication and a seamless loop dynamic.

\subsection{Phase 2A: Information gathering for Construction}

This process includes construction documentation, Tender and update of design if required. Integrated Project Delivery, or by default, Design-Build or custom variations of it are the preferred procurement option.

\subsection{Phase 2B: Construction}

The construction process itself. For this step is highly recommended the use of BIM for coordination (clash detection) and the possibility of using digital fabrication to streamline the construction process. Software like Navisworks is widely used.

\subsection{Phase 3A: Commissioning}

This step is optional, but highly recommended for verification purposes and "lessons learned". During Commissioning is when occurs the gathering of all manuals for systems operations, cleaning, inspection duties, etc.

\subsection{Phase 3B: Operations}


Facilities Management (F.M.) when the building is operating. BIM can play a key role. Literally, F.M. is not part of the design/construction process; however, it is included to provide feedback documenting lessons learned.

\section{Comparison to other models of design process}

There are several models that attempt to describe design processes, but the ones that closely resemble the current practice as observed in the sample of participants will be analyzed. Those are the AIA Best Practices: Defining the architect's basic services contributed by the AIA knowledge resources staff [5]; and the HOK Guideline for Sustainable Design [27].

According to the AIA [5], each design stage has its own set of deliverables according to a linear timeline. The reality observed in contemporary practices of sustainable design is that first, BIM enables detail of design to be similar in SD and DD, with variations of design refinement. Second, most legal agreements like the AIA contracts are widely used and attach stakeholders to a linear process, when contemporary design for sustainability is in fact more dynamic and flexible. Also, construction documentation is described as the final step in the design process in the AIA best practices, where the use of BIM enables automatic generation of documents at different scales in different stages of design.

Another example is the prescriptive method described in the HOK Guideline for Sustainable Design [27], following ten steps for an integrated design. Their ten steps have several overlaps with the description of processes found in this research. The steps seem to be tied to the AIA best practices and contractual agreements, hence described in a linear fashion that better accommodates them, despite their own design process being in reality more dynamic with the use of BIM, BES and IDP.

DEPROSU is a revised and updated model based on existent methods in the literature. The DEPROSU differentiate from existent and traditional methods in two ways: (1) some steps can have no particular order within a phase (non-linear, in opposition to the AIA linear process); and (2) is retroactive, where some steps can go back and forth emphasizing the real dynamics of the design process for sustainable architectural design, by using BIM and BES.

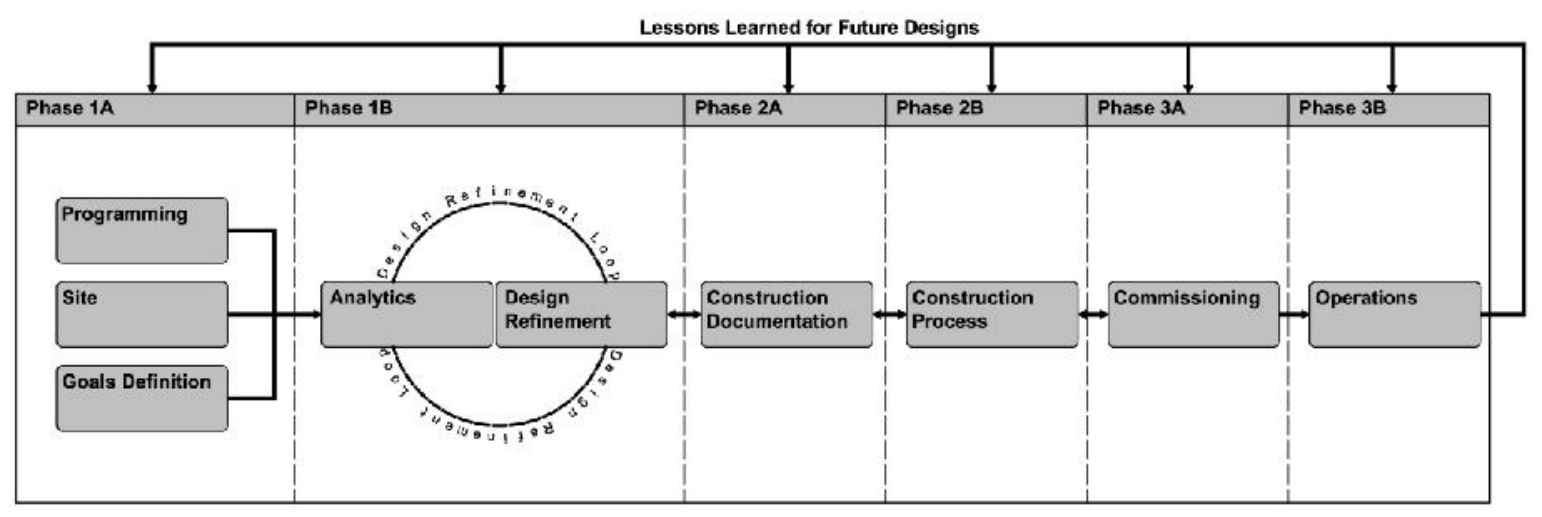

Fig. 1: Simplified diagram for DEPROSU

\section{Conclusions}

By conducting a qualitative study consisting of interviews of practitioners in leading sustainable architectural design firms, this research has produced a portrait of contemporary architectural practice. The research has confirmed the interest among practitioners in the topics of BIM, BES and IDP and the broad understanding that they are interrelated, synergistic and crucial to innovative practice. The results show that a generalization of sustainable design processes is possible. A design process for sustainability (DEPROSU) model was created by collecting best practices from data gathered from the interviews and the critical literature review. BIM and BES processes enable this method to be implemented with an integrated design team. DEPROSU includes ecological aspects of design [31], and also proposes different alternatives of software usage and normative calculations for specific design steps. DEPROSU represents an alternative design method that departs from traditional and linear processes suggested by institutionalized documents, to better reflect the dynamics of contemporary design for sustainability. To this date, this method has not been tested in a real case-scenario.

\section{Significance}

Design processes and methods are subject to radical changes that require a new class of accomplished multi-skilled professionals capable of comprehensive decision-making [33]. The significance of this research is that it provides evidence of commonalities found in the design processes of advanced sustainable architectural designers (represented in DEPROSU) and can potentially be validated as protocols or standards, providing architectural design practices with concrete patterns for improvement and or validation of their design methods. This research could guide revision and updates to contract agreements and best practices documentation. The findings from this study present a snapshot of the state of the art in 
sustainable design in 2012 and can have historical significance serving as a benchmark.

\section{Generality and limitations}

The scope of this research was to focus on the implementation of BIM, BES and IDP in sustainable design methods. The conclusions are general and valid providing valuable insight about perceptions of BIM, BES and IDP at different hierarchical levels in a sample of firms that also differs in size and scale. The conclusions deal with the design process as a whole but with an emphasis on the design process and not construction/operations. This study did not assess the effectiveness of the methods for sustainable design used in the firms; the appropriateness of the organization of each firm; the effectiveness of use of digital tools or the assessment of participants' building designs. The data set has obvious limitations and it is focused on Phase 1 of DEPROSU. The interviewees expressed their personal opinions, which could be biased based upon their professional responsibilities and might not represent the views held by others in their firms. The sample of firms is not representative of all types of design firms, and the interviewees do not represent the employees of the firms. These limitations impose constraints on the interpretation of results.

\section{Future work}

Research could explore the implementation of a generalized design process, such as the proposed DEPROSU model, in an experimental case study. The DEPROSU model could be implemented in academic situations and in professional architectural practice, including construction and operations.

Reliability of BES tools is a key component of future research. With an improved and automated processes for BES, all architectural firms could become more involved in sustainability, including small and medium firms.

Currently, the need for evidence to support design decisions is changing design methods and perhaps, the cognitive process of design. However, designers are fearful of risky experimentations due to consequences on the environment and liability. Here lies the importance of an academic research to investigate this issue in a less risky environment, whose results might serve as a base for direction of academic curricula changes.

Research could establish criteria to define training procedures on BIM and BES, to help promote in-house expertise in architecture firms. A theoretical framework for implementing training in different situations would help architectural design firms to adopt new techniques and to decide future investments.

\section{References}

[1] Pacala, S., \& Socolow, R. Stabilization wedges: Solving the climate problem for the next 50 years with current technologies. Science 13, 2004 305(5686), 968-972. DOI: $10.1126 /$ science. 1100103

[2] Pachauri, R. K., \& Reisinger, A. Contribution of Working Groups I, II and III to the Fourth Assessment Report of the Intergovernmental Panel on Climate Change. Geneva, Switzerland: IPCC, 2007

[3] Jernigan, F. E. Big BIM, little bim: The practical approach to building information modelling: Integrated practice done the right way! 1st ed. Salisbury, MD: 4Site Press, 2007.

[4] Torcellini, P., Judkoff, R., Pless, S., Deru, M., Griffith, B., \& Long, N. Lessons Learned from Case Studies of Six High-Performance Buildings (Technical Report). National Renewable Energy Laboratory. Retrieved from http://www.nrel.gov/docs/fy06osti/37542.pdf, 2006.

[5] AIA. AIA Best practices: Defining the architect's basic services contributed by the AIA knowledge resources staff, July 2007.

[6] BLISS. Building Life-Cycle information support system (BLISS). Available from http://cbs.lbl.gov/BPA/bliss.html, 1997.

[7] Fisk, P. I. Developing a design methodology for sustainable systems, 1995.

[8] Yeang, K. Ecodesign: a Manual for ecological design. Wiley and Sons, 2008.

[9] Zimmerman, A. The Integrated Design Process: Report on a national workshop. Canada, 2001.

[10] Kow, A.G. \& Grondzik, W. The Green Studio Handbook. Architectural Press, 2008.

[11] NIBS. National Building Information Model Standard. Retrieved April 27, 2009, from http://www.wbdg.org/bim/nbims.php, 2008

[12] Clayton, M., Ozener, O., Haliburton, J., \& Farías, F. (Towards studio 21: Experiments in design education using BIM. SIGraDi 2010. Presented at the Disruption, Modeling and Construction: Shifting Dialogues, Bogotá, Colombia, 2010.

[13] AIA. The business of architecture: An AIA survey report on firm characteristics. The American Institute of Architects, 2009.

[14] Smith, D., \& Tardiff, M. Building Information Modeling: A Strategic Implementation Guide for Architects, Engineers, Constructors, and Real Estate Asset Managers. Wiley, 2009.

[15] Krygiel, E., \& Nies, B. Green BIM successful sustainable design with Building Information Modelling. Wiley Publishing, 2008.

[16] Hand, J., Crawley, D., Donn, M., \& Lawrie, L. Improving non-geometric data available to simulation programs. Building and Environment, 2008, 43(4), 674-685.

[17] Knight, A., \& Ruddock, L. Advanced research methods in the built environment. Wiley-Blackwell, 2008.

[18] Oxtoby, B., McGuinness, T., \& Morgan, R.E. Developing organizational change capability. European Management Journal, 2002, 20 (3), 310-320. 
[19] CMAA Construction Management Association of America. Construction Management Standards of Practice. 10th Edition, CMAA, 2010.

[20] Vallero, D., \& Brasier, C. Sustainable design: The science of sustainability and green engineering. Wiley, 2008.

[21] Azhar, S., Carlton, W., Olsen, D., \& Ahmad, I. Building information modelling for sustainable design and LEED ${ }^{\circledR}$ rating analysis. Automation in Construction, 2011, 20, 217-224.

[22] Schlueter, A., \& Thesseling, F. Building information model based energy/exergy performance assessment in early design stages. Automation in Construction, 2009, 18(2), 153-163. DOI:10.1016/j.autcon.2008.07.003

[23] Levring, A., \& Nielsen, D. Schematic strategies and workflows for sustainable design development. Presented at the Autodesk University 2011, Las Vegas, Nevada, 2011 .

[24] Hamilton, K., Shepley, M. Design for critical care: An evidence-based approach. Architectural Press, 2009.

[25] McCullough, C. (2010). Evidence-based design for healthcare facilities. SIGMA Theta Tau International; 1 edition, 2010.
[26] Kalay, Y. The impact of information technology on design methods, products and practices. Design Studies, 2006, 27(3):357-380.

[27] Mendler, S. F., Odell, W., \& Lazarus, M. A. The HOK guidebook to sustainable design (2nd Edition). Wiley, 2005 .

[28] Peña, W., \& Parshall, S. Problem seeking: an architectural programming primer (Fourth). Wiley and Sons, 2001.

[29] Cherry, E., \& Petronis, J. Architectural Programming. Whole Building Design Guide, 2009. Retrieved from http://www.wbdg.org/design/dd_archprogramming.php

[30] Milne, M., Liggett, R., Benson, A. \& Bhattacharya, Y. Climate Consultant 4.0 [software], (2010). Available from http://www.energy-design-tools.aud.ucla.edu/

[31] Fisk, P. I. Arch 622 Seminar sustainable systems in design. Texas A\&M University, 2009.

[32] Ozener, O. Studio education for integrated practice using Building Information Modelling (PhD Dissertation). Texas A\&M University, College Station, Texas, 2009. 